\title{
The Effects of High-Intensity Interval Training on Cardiorespiratory Fitness and IL-6 in Adolescents
}

\author{
Intan Suraya Ellyas", Sugiyanto, Muhammad Furqon Hidayatullah, Muchsin Doewes, \\ Rumi Iqbal Doewes, Fadilah Umar
}

Department of Sports Science, Faculty of Sports, Sebelas Maret University, Surakarta, Central Java, Indonesia

Received March 9, 2021; Revised April 15, 2021; Accepted May 23, 2021

\section{Cite This Paper in the following Citation Styles}

(a): [1] Intan Suraya Ellyas, Sugiyanto, Muhammad Furqon Hidayatullah, Muchsin Doewes, Rumi Iqbal Doewes, Fadilah Umar , "The Effects of High-Intensity Interval Training on Cardiorespiratory Fitness and IL-6 in Adolescents," International Journal of Human Movement and Sports Sciences, Vol. 9, No. 3, pp. 568 - 576, 2021. DOI: 10.13189/saj.2021.090324.

(b): ntan Suraya Ellyas, Sugiyanto, Muhammad Furqon Hidayatullah, Muchsin Doewes, Rumi Iqbal Doewes, Fadilah Umar (2021). The Effects of High-Intensity Interval Training on Cardiorespiratory Fitness and IL-6 in Adolescents. International Journal of Human Movement and Sports Sciences, 9(3), 568 - 576. DOI: 10.13189/saj.2021.090324.

Copyright $(2021$ by authors, all rights reserved. Authors agree that this article remains permanently open access under the terms of the Creative Commons Attribution License 4.0 International License

\begin{abstract}
Cardiovascular disease (CVD), as a cause of death and illness, is increasing, which is also associated with lower levels of cardiorespiratory fitness (CRF). Physical exercise can improve CRF and spur increases IL-6 (an anti-inflammatory) induced through this same means, which therefore reduces the risk of non-communicable diseases. In addition, high-intensity interval training (HIIT) and moderate-intensity continuous training (MICT) have a positive effect on health. This study aims to compare the effect of HIIT versus MICT on CRF and the acute response to exercise-induced plasma IL-6 in healthy and untrained adolescents. Methods: An experimental study with a pre-post design was conducted on 60 untrained and healthy adolescents aged $16.1 \pm 0.4$. The groups had been trained for 6 weeks with a frequency of three days weekly, while VंO2max and blood sampling were performed before and after this activity. The CRF was tested using a multi stage fitness test (MFT), and IL-6 plasma levels were measured with ELISA methods. Results: HIIT increased CRF from $31.2 \pm 5.8$ to $35.6 \pm 6.9 \mathrm{~mL} / \mathrm{kg} / \mathrm{min}(\mathrm{p}<0.001)$ and MICT elevated $\dot{\mathrm{V} O} 2 \mathrm{max}$ from $32.3 \pm 6.8$ to $37.3 \pm 6.7 \mathrm{~mL} / \mathrm{kg} /$ $\min (\mathrm{p}<0.001)$. In fact, there was no significant difference $(p=0.292)$ between the two types of training. There was no change in the acute response of the IL- 6 at the time of the first and last exercise in both groups. Conclusion: Teenagers need to practice the HIIT and MICT training to reap the associated health benefits. The acute inflammatory response to exercise did not increase significantly, but
\end{abstract}

there were elevated levels of plasma IL-6 afterward.

Keywords Exercise, HIIT, Cytokine, VO2max, Youth

\section{Introduction}

The World Health Organization (WHO) states 31\% of deaths in the world are caused by cardiovascular disease / CVD. Physical inactivity and sedentary lifestyle cause decreased cardiorespiratory fitness (CRF) [1], which increases the risk of CVD [2]. In addition, physical inactivity is associated with metabolic syndrome (MetS) in adolescents[3]. Mets is a group of concurrent symptoms that increase the possibility of cardiovascular disease and type 2 diabetes[4]. More than two-thirds of non-communicable disease histories appear during childhood and adolescence[5]. The WHO states more than $80 \%$ of the adolescent students globally do not meet current recommendations for at least one hour of physical activity. Low cardiorespiratory fitness or low muscle strength or obesity is a strong risk factor for the appearance of psychiatric disorders, muscle diseases, and cardiovascular diseases later in life[6]. Therefore, it is a crucial intervention measure in this group of individuals to increase physical activity. Physical exercise can increase 
body mass index while reducing body fat percentage as well as having an important effect on getting the appearance of fit and agile physique[7], and enhance immunological function via a cytokine-mediated anti-inflammatory response[8]. Exercise components such as intensity, duration, and exercise volume have different effects on cytokine response[9]. Interleukin-6 is a pleiotropic cytokine that is induced by skeletal muscle contraction and enters the bloodstream[10]. Regular exercise will stimulate IL-6, which has an anti-inflammatory effect to prevent disease caused by chronic low-grade inflammation[9].

Moderate-intensity continuous training has been known for improving CRF[11], and exercise causes normal acute elevations of IL-6 and IL-10, which have anti-inflammatory effects, thereby reducing the risk of CVD and type 2 diabetes[9]. However, from several studies, high-intensity interval training also improves CRF[12]. HIIT is an extreme type of workout interspersed with rest period[13]. In recent years, a number of experimental studies have compared the effects of HIIT and MICT on CRF in children and adolescents, but the findings are inconsistent. Several studies have shown the high-intensity training increases cardiorespiratory fitness significantly compared to MICT[14]'[15]'[16], while others did not observe differences between the two methods[17]. There is evidence that HIIT is also beneficial for children and adolescents, with the ability to reduce fat[14] and increase the composition of the body effectively[18]. Several exercise methods have been devised to make physical exercise a fun activity[19] and more attractive aimed at improving adherence among adolescents, and adolescents often do not follow the recommended guidelines for regularly engaging in moderate-intensity exercise for 30-60 minutes a day[20]. Therefore, it is necessary to draw up an exercise program with the aim of improving aerobic fitness that has a high level of compliance. High-intensity interval training (HIIT) has shown good adherence among adolescents[21]

Some studies focused on the obese population [22]. However, not many have performed it in the normal weight group that is also untrained. Therefore, further research needs to be conducted on complex interval training variables with modifications on the work, rest, and type of exercise utilized. This study aims to compare the effects of HIIT training with MICT on aerobic/cardiorespiratory fitness (CRF) and the acute response to exercise-induced IL-6 in healthy and untrained adolescents.

\section{Materials and Methods}

Subjects were 60 (male $\mathrm{n}=30$; female $\mathrm{n}=30$ ) healthy, and untrained adolescents, who were recruited from SMA Pradita Dirgantara, Boyolali, Indonesia. All subjects have lived in the school dormitory for three months. In this study, subjects had the same daily activity schedule, diet, and types of activities. The training implementation is done after the mid-semester exam and before the semester exam so that it does not interfere with the learning process. The research subjects fulfilled the inclusion criteria were as follows: (i) adolescent aged 15-18; (ii) body mass index (BMI) 18-30 kg.m-2; (iii) blood pressure 90/60 mmHg 140-90 mmHg; (iv) untrained (do not follow the exercise more than one week of high intensity); (v) not take vitamin supplements or anti-inflammatory drugs. The exclusion criteria were; (i) smoking; (ii) musculoskeletal disorders; (iii) have a chronic disease. 81 participants entered the inclusion criteria (male $\mathrm{n}=37$; female $=51$ ). Participants were taken using random quota sampling to 30 males and 30 females. Each sex group was then divided into HIIT and MICT treatment according to aerobic fitness level by ordinal pairing method.

The participants presented a health and neuromuscular status that ensures their ability to complete the study protocol. Written informed consent was obtained from all subjects after they had been informed about the purpose and risks of the study and also following a routine medical screening. The parents and the principal signed the informed consent letters. All procedures of this study were approved by the Dr. Moewardi General Hospital Health Research Ethics Committee in letter No.1.131/X/HREC/2019 dated October 4, 2019. The sample size was calculated using the G*Power 3 Software [23]. We used both delta changes and SD from previous studies of a similar exercise intervention extension, where IL-6 was included [24]. Based on this, with one predictive outcome (IL-6 [SD: $19 \mathrm{pg} / \mathrm{mL}]$ ), a moderate effect size $(0.55)$ and a critical $t$ value of 1.29 , a total sample size of (n $=30$ ) subjects per group would give a statistical power of $80 \%$, under an alpha error $\mathrm{p}<0.1$. The characteristics of the subjects are listed in table 1 . One participant in the MICT group engaged in the complete exercise but was unable to take the final MFT test due to an unfamiliar pain. The flow chart diagram is detailed in Figure 1.

Table 1. Characteristic of the subjects before training

\begin{tabular}{|c|c|c|}
\hline Characteristic & \multicolumn{2}{|c|}{ Exercise Methods } \\
\hline & $\begin{array}{c}\text { HIIT } \\
(\mathbf{n}=\mathbf{3 0})\end{array}$ & $\begin{array}{c}\text { MICT } \\
(\mathbf{n}=\mathbf{3 0})\end{array}$ \\
\hline Age (years) & $16,2 \pm 0,4$ & $16,00 \pm 0,4$ \\
\hline Height (m) & $165,3 \pm 6,5$ & $161,5 \pm 8,5$ \\
\hline Weight $(\mathrm{kg})$ & $55,4 \pm 9,2$ & $52,8 \pm 11,7$ \\
\hline BMI (kg.m $\left.{ }^{-2}\right)$ & $20,4 \pm 3,0$ & $20,2 \pm 4,3$ \\
\hline Hb (g/dL) & $15,3 \pm 1,0$ & $13,1 \pm 1,3$ \\
\hline Sistolik BP (mmHg) & $105,4 \pm 9,2$ & $109,47 \pm 9,8$ \\
\hline Diastolik BP (mmHg) & $67,0 \pm 7,0$ & $69,5 \pm 8.6$ \\
\hline VंO2max (mL/kg/min) & $31,2 \pm 5,8$ & $32,3 \pm 6,8$ \\
\hline IL-6 (pg/mL) & $93,7 \pm 80,6$ & $65,0 \pm 50,15$ \\
\hline
\end{tabular}

All values are presented as mean $\pm \mathrm{SD}$. HIIT=high-intensity interval training; $\mathrm{MICT}=$ moderate-intensity continuous training. 


\subsection{Measurement of Cardiorespiratory Fitness}

All participants had received an explanation about the study one week earlier. Meanwhile, three, prior to the commencement, physical examination, anthropometric, and initial CRF measurements, were performed. The CRF was conducted using a multistage fitness test (MFT). MFT is a valid and reliable test for $\mathrm{V} O 2$ max prediction in men and women [25]. Subjects were given a warm-up 5 minutes before MFT was measured. The subject performs a $20 \mathrm{~m}$ back and forth run according to the rhythm of the tape. Level achievement is considered a failure (maximum level) if the subject fails to reach the distance limit of $20 \mathrm{~m}$ twice. Level achievement matched with $\dot{V} O 2$ max value prediction table then matched with aerobic fitness norm table [25]. MFT for examining the effect of the training was carried out two days after the last exercise. The study protocol is shown in Figure 2.

\subsection{Study Design}

The training was conducted three times a week, with a break of 1-2 days before the next. The exercise is done in the morning and at the same time, starting at 5 in the morning. Exercises are carried out in conditions after a night of fasting (i.e., no breakfast was consumed). All training sessions were monitored by a qualified trainer using PolarRS800CX GPS. The maximum heart rate (MHR) was calculated through measurements of 208-0.7 participants [26]

\subsubsection{High-Intensity Interval Training (HIIT)}

The subjects performed intermittent run (total duration, 25-30min), where each exercise consisted of two sets with $4 \times 30$ seconds per one, and the intensity of $80-95 \%$ maximum heart rate (MHR). Furthermore, a 1:3 work $/$ recovery ratio of $60-70 \%$ MHR breaks between the sets was used. The exercise applied the principle of progression by increasing repetition from four times in the first and second weeks, to five in the third and fourth, and six times in the fifth and sixth. The subjects warmed up before training for 5 minutes and rested for the same period after the completion. The description of 6 weeks of HIIT and MICT are listed in table 2.

\subsubsection{Moderate-intensity continuous training (MICT)}

The subjects performed 40 minutes run continuously at $60-75 \%$ MHR, while the general warm-up was at $50 \%$ for five minutes. The exercise applied the principle of progression by increasing time from forty minutes in the first and second weeks, to fifty in the third and fourth, and sixty in the fifth and sixth. Cooling for 5 minutes is done after finishing the exercise.

Table 2. Description of 6 weeks of HIIT and MICT

\begin{tabular}{|c|c|c|}
\hline & HIIT & MICT \\
\hline Frequency & 3 times/week & 3 times/week \\
\hline Duration & $20-25$ min & $40-60$ min \\
\hline Intensity & $\begin{array}{c}\text { Work }: \text { Recovery Ratio } \\
=1: 3 \\
\text { Work }\end{array}$ & \\
& $\begin{array}{c}80-95 \% \text { HRmax } \\
\text { Relief }=\end{array}$ & $60-75 \%$ MHR \\
$60-70 \%$ HRmax & \\
\hline $\begin{array}{c}\text { Type of } \\
\text { activity }\end{array}$ & $\begin{array}{c}\text { Interval running } \\
\text { Continuous running }\end{array}$ \\
\hline
\end{tabular}

HIIT=high-intensity interval training; MICT=moderate-intensity continuous training.

\subsubsection{Blood Sampling and examination}

Venous blood samples $(10 \mathrm{ml})$ were obtained before and immediately after each exercise in the first training and at the end of week 6 . Also, they were instantly allocated into two $5 \mathrm{ml}$ vacutainer tubes containing EDTA for plasma separation and blood analysis. This was centrifuged at $1.000 \mathrm{~g}$ and $40 \mathrm{C}$ for 15 minutes, and then the supernatant was stored at $800 \mathrm{C}$ for future use. The concentration of IL-6 was analyzed by ELISA commercial kits (Komabiotech Human IL-6) on a microplate reader iMark Biorad device.

\subsection{Statistical Analysis}

Participants were placed into matched groups based on VंO2max: a HIIT group and a MICT group using ordinal pairing methods so that each group consisted of the same aerobic fitness level. Before a comparative analysis of changes in $\dot{\mathrm{V} O} 2 \mathrm{max}$ and plasma IL-6 levels was carried out, a data normality test was also performed to determine the appropriate method. When the data were normally distributed, a paired and independent sample t-test was used. However, when it wasn't, non-parametric Wilcoxon and Mann Whitney analysis was used. Statistical significance was set at $5 \%$ for all analyses, and the calculations were conducted using SPSS, version 22. 


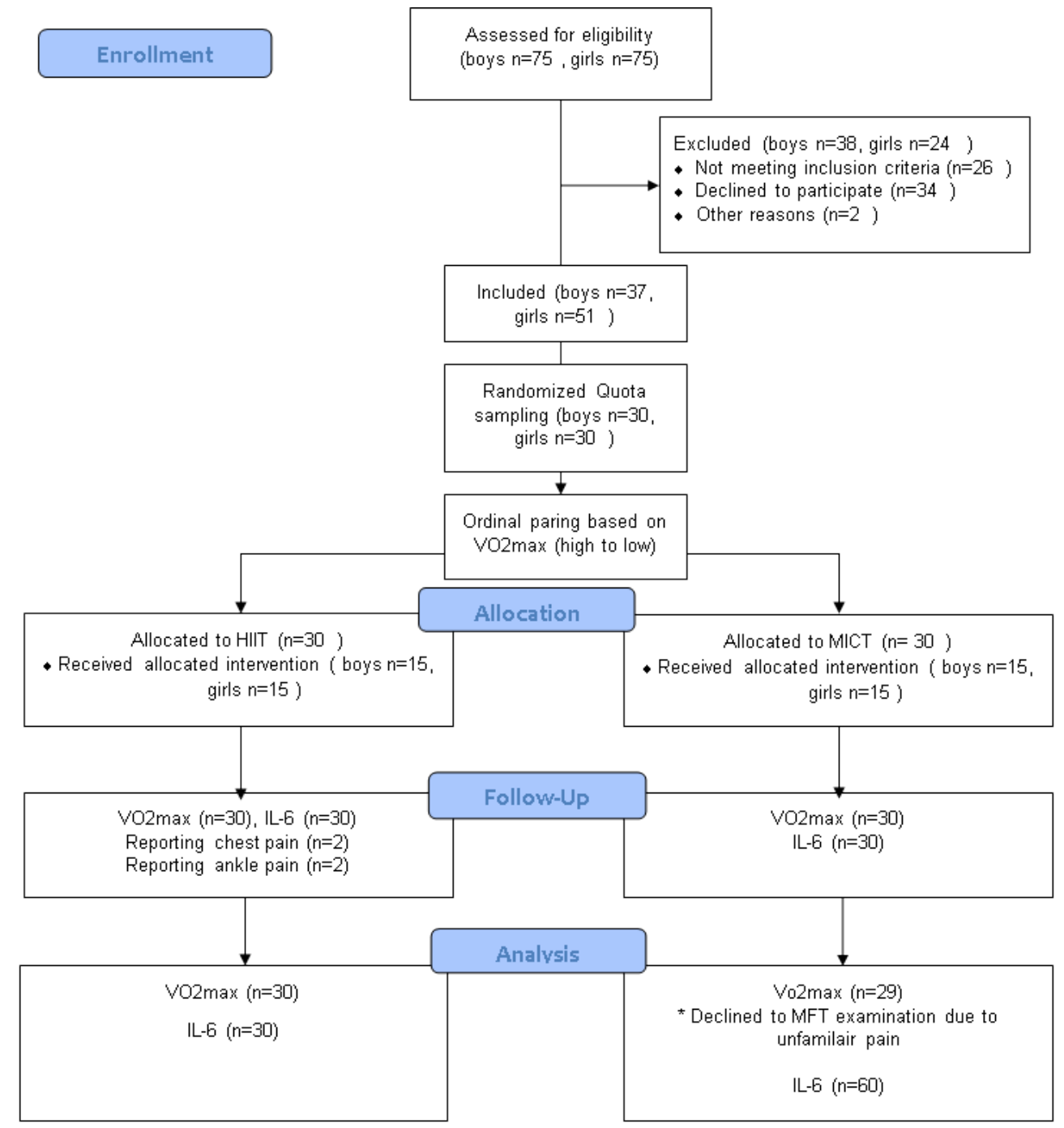

Figure 1. Flow-chart diagram

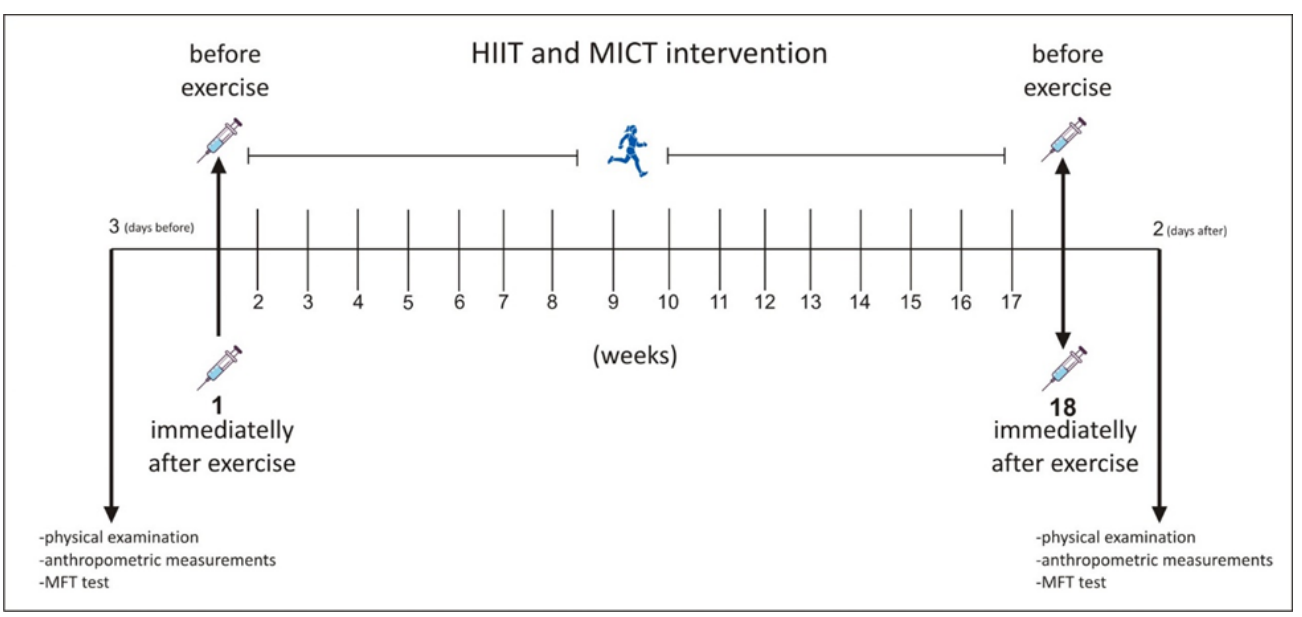

Figure 2. Study Protocol 


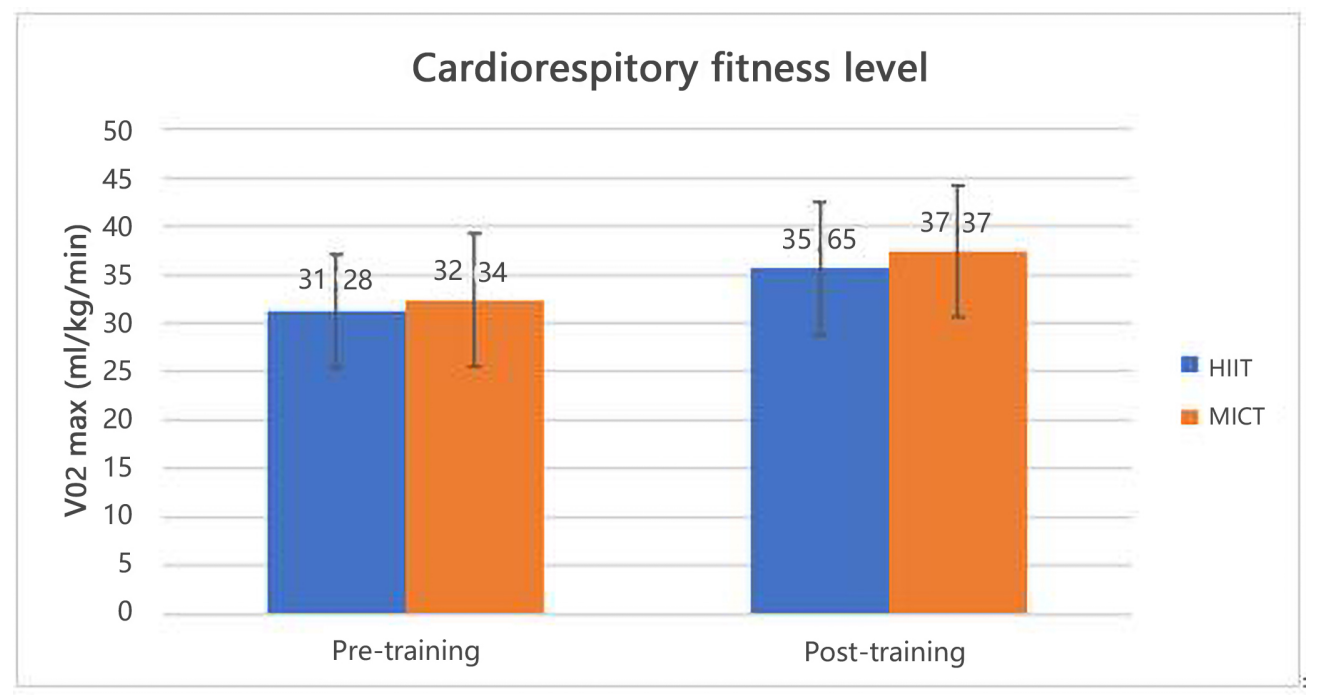

Figure 3. Cardiorespiratory fitness (CRF) before and after the training period. Data distributed normally. In the HIIT group, there was a significant difference with the increase in CRF ( $p<0,001)$; in the MICT group, there was also an increase in CRF $(\mathrm{p}<0,001)$. No significant differences found between-group $(\mathrm{p}=0$,292) HIIT=high-intensity interval training; MICT=moderate-intensity continuous training.

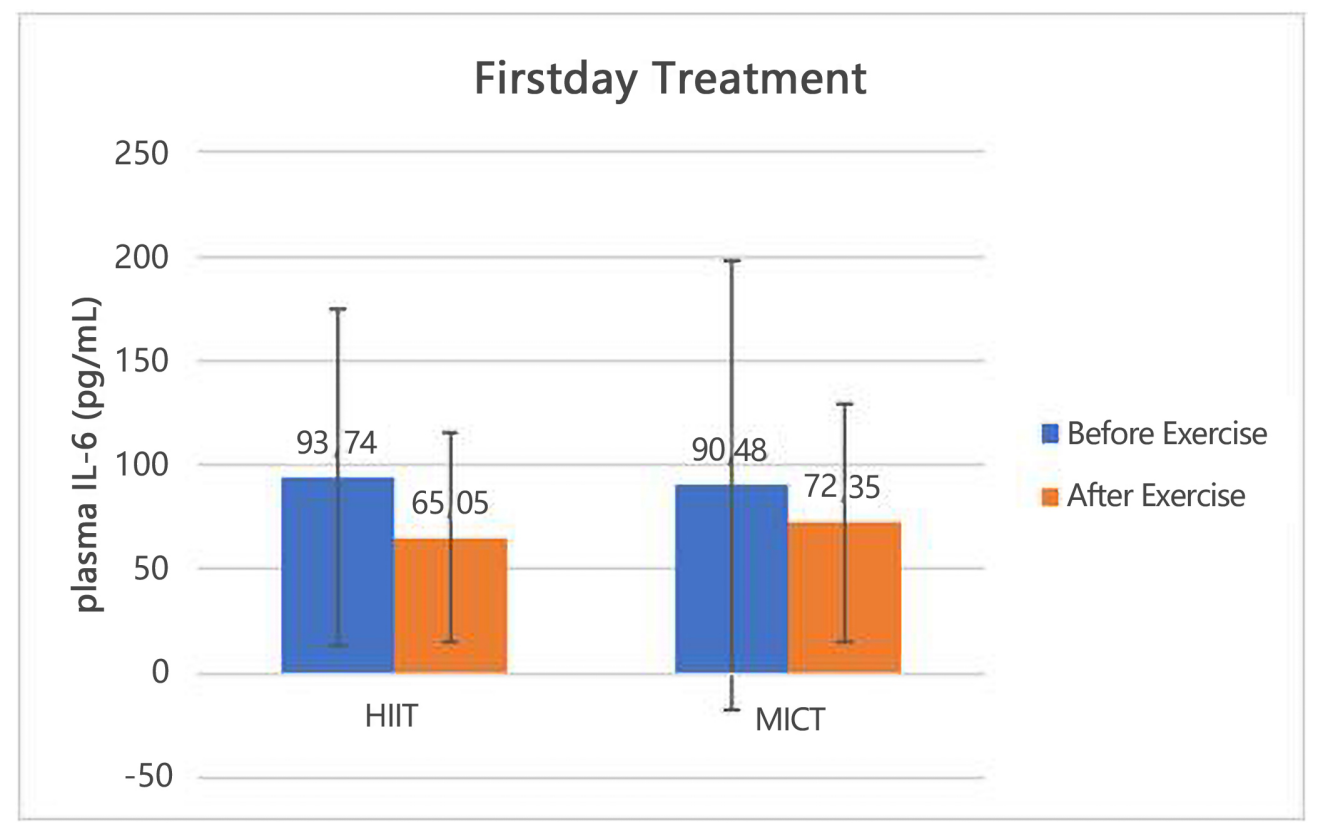

Figure 4. IL- 6 response to exercise before and after exercise at first-day training. The HIIT group experienced a decrease in IL-6 ( $p=0,845$ ); the MICT group experienced an increase $(\mathrm{p}=0,846)$. No significant differences were found between HIIT dan MICT $(\mathrm{p}=0,912)$ on first-day training. IL-6=interleukin-6; HIIT=high-intensity interval training; MICT=moderate-intensity continuous training. 


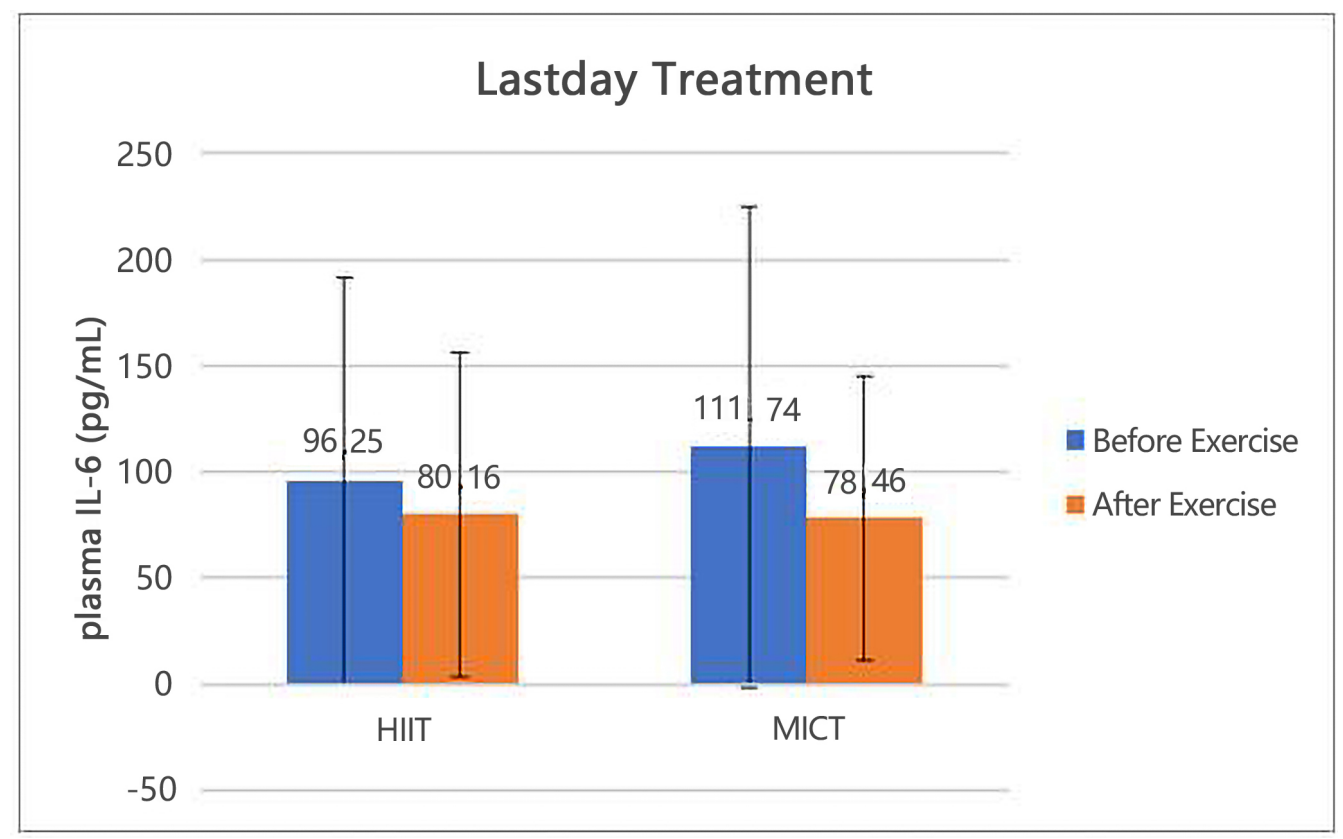

Figure 5. IL-6 response to exercise before and after exercise at last day training. The HIIT group experienced an increase ( $\mathrm{p}=0,178)$, but the MICT group experienced a decrease $(\mathrm{p}=0,704)$. No significant differences were found between HIIT and MICT $(\mathrm{p}=0,255)$. The mean increase in the HIIT group was higher than MICT. IL-6=interleukin-6; HIIT=high-intensity interval training; MICT=moderate-intensity continuous training.

\section{Results}

Data were presented as mean value \pm standard deviations (SD). The characteristics of the research subject are listed in Table 1. Those who were studied were adolescents consisting of 30 males and 30 females aged $16.1 \pm 0.4$ with normal composition, that is, mean body mass index (BMI) of less than $25 \mathrm{~kg} . \mathrm{m}-2$. Hemoglobin levels were also normal in the two exercise groups. In addition, cardiorespiratory fitness (CRF) was represented as $\dot{\mathrm{V} O} 2 \mathrm{max}$, while the results below $38 \mathrm{~mL} / \mathrm{kg} / \mathrm{min}$ in both of them indicated that the study subjects were untrained[27].

Participants' $\dot{\mathrm{V} O} 2 \mathrm{max}$ before and after the training were presented in figure 3 . The analysis results using the paired samples test showed significant differences in the two groups. Those on HIIT showed a significant increase in the mean $\dot{\mathrm{VO}} 2 \mathrm{max}$, which was $31.2 \pm 5.8 \mathrm{ml} / \mathrm{kg} / \mathrm{min}$ before exercise and was $35.5 \pm 6.9 \mathrm{ml} / \mathrm{kg} / \mathrm{min}$ after $(\mathrm{p}$ $<0.001)$. The mean $\dot{V} O 2$ max in the participants on MICT before training was $32.3 \pm 6.8 \mathrm{ml} / \mathrm{kg} / \mathrm{min}$, which increased to $37.3 \pm 6.7 \mathrm{ml} / \mathrm{kg} / \mathrm{min}(\mathrm{p}<0.001)$. The changes that occurred in the HIIT group was not significantly different from those in the MICT group $(\mathrm{p}=0.292)$.

There were abnormal data in the normality test of IL-6 plasma levels; hence non-parametric Wilcoxon and Mann Whitney were used for the analysis. Acute response to the interleukin-6 levels occurred due to exercise on the first day of the training. It was known that the average plasma level of this anti-inflammatory agent in the HIIT group before exercise (pre-test) was $93.74 \mathrm{pg} / \mathrm{mL}$, but decreased after to $90.48 \mathrm{pg} / \mathrm{mL}$ which was not statistically significant $(\mathrm{p}=0.845)$. However, for the MICT group, the pre-test average was $65.05 \mathrm{pg} / \mathrm{mL}$; an increase to 72.35 $\mathrm{pg} / \mathrm{mL}$ occurred after the exercise, which was insignificant $(p=0.846)$. Comparison of the acute changes in both training groups on the first day of exercise was not significantly different $(p=0.912)$. It is explained in figure 4.

On the last day, there was an acute response to plasma IL-6 levels that occurred due to exercise. It was known that the average levels of this anti-inflammatory agent in the HIIT group before exercise (pre-test) were 96.25 $\mathrm{pg} / \mathrm{mL}$, which increased after to $111.74 \mathrm{pg} / \mathrm{mL}$ insignificantly $(p=0.178)$. However, for the MICT group, the pre-test average was $80.16 \mathrm{pg} / \mathrm{mL}$, which decreased after the exercise to $78.46 \mathrm{pg} / \mathrm{mL}$ insignificantly $(\mathrm{p}=$ 0.704). Comparing the acute changes that occurred in both groups on the last day of training was not significantly different $(p=0.255)$. It is explained in figure 5 . The average increase in HIIT is higher than in the MICT. There was an insignificant difference in acute response to plasma IL-6 levels between the first exercise and the last in the HIIT and MICT groups at $\mathrm{p}=0.209$ and $\mathrm{p}=0.506$, respectively.

\section{Discussion}

The main finding was that the 6-week HIIT and MICT protocol improved the $\dot{\mathrm{V} O} 2 \mathrm{max}$. The other findings were that there was an insignificant difference in acute response to plasma IL-6 levels between the first exercise and the last in the HIIT and MICT groups at $p=0.209$ and $p=$ 0.506 , respectively. The increase in the IL- 6 means acute response was higher in the HIIT than in the MICT group 
at the end of the exercise, which was insignificantly different $(\mathrm{p}=0.255)$.

After the six weeks of training, participants in both study groups had marked increases in $\dot{\mathrm{V} O} 2 \mathrm{max}$. Figure 3 explains different exercise methods have the ability to increase aerobic capacity. $\dot{\mathrm{V} O} 2 \mathrm{max}$ is an indicator of total cardiorespiratory fitness and oxygen transport to the respiratory, cardiovascular, and muscular systems in order to enhance supply to the mitochondria for oxidative metabolism. In addition, both methods increased this variable significantly, and there was no difference between them. Therefore, it was stated that HIIT is as good as MICT in elevating CRF in healthy adolescents.

The results obtained are consistent with the study by Alonso-Fernandez [28] that carried out the HIIT program using the Tabata method on training for seven weeks to increase $\mathrm{V} O 2 m a x$ in adolescents. The study conducted by Racil et al.[29] also showed improvements in this particular variable with the high-intensity training in obese adolescent girls. In other contexts with different training modes, it was shown that HIIT is greater in reducing risk factors for cardiovascular disease than moderate-intensity exercise[21]. Other research conducted on soccer athletes under the age of 18 years there is an increase in $\dot{\mathrm{V} O} 2 \mathrm{max}$ using basic soccer training[30]. Increased $\dot{\mathrm{V} O} 2 \mathrm{max}$ in the subjects is important because the improvement is associated with a reduced CVD risk. HIIT, with the advantage of being short in time[15] and requiring no space or long distances, has some benefits over the continuous method; it is also more efficient in improving body composition and rating of perceived exertion (RPE) after maximal level exercise[20]. HIT exercise method is a decent and time-efficient exercise to improve cardiorespiratory fitness and body composition in the adolescent population[21].

MacInnis \& Gibala (2017) stated that HIIT increases the oxidative capacity of human skeletal muscle by various biochemical mechanisms in mitochondrial respiration in the form of maximum activity or total protein content. The bioenergy from HIIT depends on the duration and intensity of the number of intervals performed and the duration of recovery. It is unique in HIIT due to the presence of cellular energy, which is dominated by non-oxidative or oxidative systems[31].

The intensity of the exercise and the pattern of contractions are important determinants of human skeletal muscle remodeling. MacInnis et al.[32] stated that HIIT has the ability to increase the maximal activity of citrate synthase higher than MICT. Similarly, interval training is thought to stimulate different energy pathways from MICT in mitochondrial biogenesis[33]. HIIT also increases the capacity of aerobic energy metabolism in the form of physiological improvements due to changes obtained from high volume MICT[34]. In addition, studies carried out by Kilpatrick et al.[34], and Milanović et al.[15] also showed that a higher response to CRF increase was found in HIIT training compared to MICT.

The second finding of this study was that there was no significant difference in acute response to plasma IL-6 levels at the first and last six weeks of exercise in the HIIT or MICT groups. However, in those on high-intensity training, there was an increase in this anti-inflammatory agent at the end of the activity. Meanwhile, the counterpart experienced a decrease. The study suggested that the two methods have different effects on inflammatory markers in healthy adolescents. Meanwhile, the increase discovered in the HIIT group explains that IL-6 is triggered by exercise and peaks at the end of the activity or show early recovery in accordance with the literature of the study [5]. Exercise intensity affects IL-6 levels[15], which increases proportionally with the response. Previous studies on the effects of HIIT programs on the total amount of this anti-inflammatory agent in the plasma have been conducted, but the results were inconsistent. In the study conducted by Barry et al.[35], HIIT and MICT exercise did not affect concentrations of IL-6 in plasma. In this study, the acute IL-6 response in the MICT group during the last exercise decreased. It is likely that this results from the normal process of adaptation of the exercise. IL- 6 levels are influenced by glycogen levels; in people who are trained, glycogen levels will increase and affect the repair of beta-oxidant enzymes thereby improving fat oxidation[9]. In contrast to others, high-intensity training increased the IL-6 levels immediately after exercise, and the HIIT response was higher than the counterpart[33]. There was manipulation on this variable, namely the length of work and rest duration, even though the same method/subject can produce different adaptation reactions. During the exercise, IL-6 was the first cytokine detected to be released by muscles into the bloodstream[6], while the target tissues are the liver, pancreas, and fat. Acute IL-6 signaling increases hepatic glucose production, causes lipolysis, and elevates the viability of pancreatic beta cells and insulin secretion. Intramuscular IL-6 enhances the positive effects of glucose uptake and fat oxidation via the PI3K and AMPK6 signaling pathways. In the acute response, the elevation in IL-6 was driven by the inhibition of carbohydrate utilization that accompanies glycogen depletion. Considering HIIT, it causes the greatest glycogen reduction and acute increases in inflammation, which reduces the chronic cases; thus, this exercise is very useful[36]. The IL-6 increased due to the effect of the high-intensity training, which is an anti-inflammatory that stimulates the production of cytokines such as IL-1 receptor antagonist and IL-10. This also suppresses TNF- $\alpha$ through the dependent and independent IL-6 pathways[37]. Contracted muscles produce and secrete Interleukin- 6 into the circulation, which has a positive effect on health, namely the regulation of glucose homeostasis by affecting gastric emptying, and actively controls the visceral adipose tissue 
mass in humans[36].

The results of this study allow the HIIT method to have a positive effect on adolescents considering that it is becoming a trend of the preferred type of exercise in the last decade, especially among young people. The HIIT method becomes a promising exercise option as a time-efficient exercise method to produce similar or greater CRF enhancements compared to MICT[38]. The results of the study in the student group recommended lifestyle changes in this group to increase physical activity especially the time and intensity of exercise to achieve good health status[39]. Actually, the research subjects were healthy adolescents. Meanwhile, most of the previous studies used overweight or obese individuals; thus, the HIIT method is also useful in those that are healthy. HIIT can be applied to other age groups, namely children, adults, and the elderly, with modification of exercise variables according to physical condition[13],

Overall, the strengths of this study are that the subjects live in school dormitories. Thus, their diet, rest time, or daily activities at school can be controlled. Participants before and during exercise did not take supplements. Therefore, the results were not influenced by these factors. The type of training was running. This type of running was easier to do, does not need special skills, and can be done by adolescents. However, the limitation was that it measured only one type of cytokine, which was not a clearer picture of the pro-inflammatory effects of exercise.

\section{Conclusions}

In conclusion, six weeks of HIIT and MICT training in healthy and untrained adolescents improved $\dot{\mathrm{V} O} 2 \mathrm{max}$ and had a positive effect on metabolic adaptation and inflammation through the release of exercise-induced cytokines. The shorter time of the high-intensity method is an option for adolescents as an effort to reduce cardiovascular risk factors.

\section{Acknowledgements}

The authors are grateful to Intan Savira and Pandu Setiawan for their assistance in collecting data for this study and the participants for their consistent effort.

\section{REFERENCES}

[1] S. Kodama et al., "Cardiorespiratory fitness as a quantitative predictor of all-cause mortality and cardiovascular events in healthy men and women: A meta-analysis," JAMA - J. Am. Med. Assoc., vol. 301, no. 19, pp. 2024-2035, May 2009, doi: 10.1001/jama.2009.681.

[2] H. W. Kohl et al., "The pandemic of physical inactivity:
Global action for public health," Lancet, vol. 380, no. 9838, pp. 294-305, 2012, doi: 10.1016/S0140-6736(12)60898-8.

[3] R. G. De Oliveira and D. P. Guedes, "Physical activity, sedentary behavior, cardiorespiratory fitness and metabolic syndrome in adolescents: Systematic review and meta-analysis of observational evidence," PLoS One, vol. 11, no. 12, pp. 1-24, 2016, doi: 10.1371/journal.pone.0168503.

[4] P. W. F. Wilson, R. B. D’Agostino, H. Parise, L. Sullivan, and J. B. Meigs, "Metabolic syndrome as a precursor of cardiovascular disease and type 2 diabetes mellitus," Circulation, vol. 112, no. 20, pp. 3066-3072, 2005, doi: 10.1161/CIRCULATIONAHA.105.539528.

[5] L. Guariguata and S. Jeyaseelan, "Children and Non Communicable Global Burden Report 2019,” 2019. [Online]. Available: www.ncdchild.org.

[6] P. Henriksson et al., "Fit for life ? Low cardiorespiratory fitness in adolescence is associated with a higher burden of future disability," $\mathrm{Br} J$ Sport. Med, pp. 1-2, 2020, doi: 10.1136/bjsports-2020-102605.

[7] N. Yaman, "The Physical Effects of the Fitness Exercises," Int. J. Hum. Mov. Sport. Sci., vol. 4, no. 3, pp. 33-38, 2016, doi: 10.13189/saj.2016.040301.

[8] B. K. Pedersen and M. A. Febbraio, "Muscle as an Endocrine Organ: Focus on Muscle-Derived Interleukin-6," Physiol. Rev., vol. 88, no. 4, pp. 1379-1406, Oct. 2008, doi: 10.1152/physrev.90100.2007.

[9] B. K. Pedersen, "Anti-inflammatory effects of exercise: role in diabetes and cardiovascular disease," Eur. J. Clin. Invest., vol. 47, no. 8, pp. 600-611, 2017, doi: 10.1111/eci.12781.

[10] M. Ost, V. Coleman, J. Kasch, and S. Klaus, "Regulation of myokine expression: Role of exercise and cellular stress," Free Radic. Biol. Med., vol. 98, pp. 78-89, 2016, doi: 10.1016/j.freeradbiomed.2016.02.018.

[11] G. Morici, C. I. Gruttad'Auria, P. Baiamonte, E. Mazzuca, A. Castrogiovanni, and M. R. Bonsignore, "Endurance training: Is it bad for you?," Breathe, vol. 12, no. 2, pp. 140-147, 2016, doi: $10.1183 / 20734735.007016$.

[12] C. Cabral-santos et al., "Similar Anti-Inflammatory Acute Responses from Moderate-Intensity Continu- ous and High-Intensity Intermittent Exercise," no. October, pp. 849 $856,2015$.

[13]K. S. Weston, U. Wisløff, and J. S. Coombes, "High-intensity interval training in patients with lifestyle-induced cardiometabolic disease: A systematic review and meta-analysis," Br. J. Sports Med., vol. 48, no. 16, pp. 1227-1234, 2014, doi: 10.1136/bjsports-2013-092576.

[14]D. Alonso-Fernández, R. Fernández-Rodríguez, Y. Taboada-Iglesias, and Á. Gutiérrez-Sánchez, "Impact of a HIIT protocol on body composition and VO2max in adolescents," Sci. Sports, vol. 34, no. 5, pp. 341-347, Oct. 2019, doi: 10.1016/j.scispo.2019.04.001.

[15]Z. Milanović, G. Sporiš, and M. Weston, "Effectiveness of High-Intensity Interval Training (HIT) and Continuous Endurance Training for VO2max Improvements: A Systematic Review and Meta-Analysis of Controlled Trials," Sport. Med., vol. 45, no. 10, pp. 1469-1481, 2015, doi: 10.1007/s40279-015-0365-0. 
[16]L. Su et al., "Effects of HIIT and MICT on cardiovascular risk factors in adults with overweight and/or obesity: A meta-analysis," PLoS One, vol. 14, no. 1, p. e0210644, Jan. 2019, doi: 10.1371/journal.pone.0210644.

[17] V. H. Arboleda-serna, Y. Feito, F. A. Patiño-villada, and A. Viviana, "Effects of high-intensity interval training compared to moderate-intensity continuous training on maximal oxygen consumption and blood pressure in healthy men: A randomized control ... Effects of high-intensity interval training compared to moderate-inte," no. October, 2019, doi: 10.7705/biomedica.4451.

[18] S. A. Costigan, N. Eather, R. C. Plotnikoff, D. R. Taaffe, and D. R. Lubans, "High-intensity interval training for improving health-related fitness in adolescents: a systematic review and meta-analysis.," Br. J. Sports Med., vol. 49, no. 19, pp. 12531261, Oct. 2015, doi: 10.1136/bjsports-2014-094490.

[19]G. B., S. T., and U. A., "The Effects of Aerobics Exercise Programmes on Body Composition and Some Physical Parameters for the Pre-obese Class 1 Obese Students at High School Aged 15-17," Int. J. Hum. Mov. Sport. Sci., vol. 4, no. 3, pp. 39-44, 2016, doi: 10.13189/saj.2016.040302.

[20] B. Bond et al., "Exercise Training in Cardiovascular Disease : Mechanisms and Two weeks of high-intensity interval training improves novel but not traditional cardiovascular disease risk factors in adolescents," no. 19, pp. 1039-1047, 2021, doi: 10.1152/ajpheart.00360.2015.

[21] S. A. Costigan, N. Eather, R. C. Plotnikoff, D. R. Taaffe, and D. R. Lubans, "High-intensity interval training for improving health-related fi tness in adolescents: a systematic review and meta-analysis," no. April 2014, pp. 1-9, 2015, doi: 10.1136/bjsports-2014-094490.

[22] A. García-Hermoso, A. J. Cerrillo-Urbina, T. Herrera-Valenzuela, C. Cristi-Montero, J. M. Saavedra, and V. Martínez-Vizcaíno, "Is high-intensity interval training more effective on improving cardiometabolic risk and aerobic capacity than other forms of exercise in overweight and obese youth? A meta-analysis," Obes. Rev., vol. 17, no. 6, pp. 531-540, 2016, doi: 10.1111/obr.12395.

[23]F. Faul, E. Erdfelder, A.-G. Lang, and A. Buchner, "G*Power 3: A flexible statistical power analysis program for the social, behavioral, and biomedical sciences," Behav. Res. Methods, vol. 39, no. 2, pp. 175-191, 2007, doi: https://doi.org/10.3758/BF03193146.

[24] L. Cipryan, "IL-6, Antioxidant Capacity and Muscle Damage Markers Following High-Intensity Interval Training Protocols," J. Hum. Kinet., vol. 56, no. 1, pp. 139-148, 2017, doi: 10.1515/hukin-2017-0031.

[25] G. Raghuveer et al., "Cardiorespiratory Fitness in Youth: An Important Marker of Health: A Scientific Statement from the American Heart Association," Circulation, pp. E101-E118, 2020, doi: 10.1161/CIR.0000000000000866.

[26] H. Tanaka, K. D. Monahan, and D. R. Seals, “Age-predicted maximal heart rate revisited," J. Am. Coll. Cardiol., vol. 37, no. 1, pp. 153-156, 2001, doi: 10.1016/S0735-1097(00)010 54-8.

[27] G. R. Tomkinson et al., "International normative $20 \mathrm{~m}$ shuttle run values from 1142026 children and youth representing 50 countries," Br. J. Sports Med., vol. 51, no. 21, pp. 1545-1554, 2017, doi: 10.1136/bjsports-2016-095987.

[28] D. Alonso-Fernández, R. Fernández-Rodríguez, Y. Taboada-Iglesias, and Á. Gutiérrez-Sánchez, "Impact of a HIIT protocol on body composition and VO2max in adolescents," Sci. Sports, vol. 34, no. 5, pp. 341-347, Oct. 2019, doi: 10.1016/j.scispo.2019.04.001.

[29] G. Racil et al., "Greater effects of high-compared with moderate-intensity interval training on cardio-metabolic variables, blood leptin concentration and ratings of perceived exertion in obese adolescent females," Biol. Sport, vol. 33, no. 2, pp. 145-152, 2016, doi: 10.5604/20831862.1198633.

[30] R. Bahtra, M. Asmawi, Widiastuti, and F. Dlis, "Improved vo2max: The effectiveness of basic soccer training at a young age," Int. J. Hum. Mov. Sport. Sci., vol. 8, no. 3, pp. 97-102, 2020, doi: 10.13189/saj.2020.080304.

[31] M. Gibala, "Physiological adaptations to low-volume high intensity interval training," Sport. Sci. Exch., vol. 28, no. 139, pp. 1-6, 2015.

[32] M. J. MacInnis and M. J. Gibala, "Physiological adaptations to interval training and the role of exercise intensity," $J$. Physiol., vol. 595, no. 9, pp. 2915-2930, May 2017, doi: 10.1113/JP273196.

[33] J. P. Little, A. Safdar, D. Bishop, M. A. Tarnopolsky, and M. J. Gibala, "An acute bout of high-intensity interval training increases the nuclear abundance of PGC-1 $\smile$ and activates mitochondrial biogenesis in human skeletal muscle," Am J Physiol Regul Integr Comp Physiol, no. 31, pp. 1303-1310, 2011, doi: 10.1152/ajpregu.00538.2010.

[34] M. J. Gibala, J. B. Gillen, and M. E. Percival, "Physiological and Health-Related Adaptations to Low-Volume Interval Training: Influences of Nutrition and Sex," Sport. Med., vol. 44, pp. 127-137, 2014, doi: 10.1007/s40279-014-0259-6.

[35] J. C. Barry, S. Simtchouk, C. Durrer, M. E. Jung, A. L. Mui, and J. P. Little, "Short-term exercise training reduces anti-inflammatory action of interleukin-10 in adults with obesity," Cytokine, vol. 111, no. June, pp. 460-469, 2018, doi: 10.1016/j.cyto.2018.05.035.

[36] D. J. Elmer, R. H. Laird, M. D. Barberio, and D. D. Pascoe, "Inflammatory, lipid, and body composition responses to interval training or moderate aerobic training," Eur. J. Appl. Physiol., vol. 116, no. 3, pp. 601-609, Mar. 2016, doi: $10.1007 / \mathrm{s} 00421-015-3308-4$.

[37] M. W. Kilpatrick, M. E. Jung, and J. P. Little, "High-intensity interval training: A review of physiological and psychological responses," ACSM's Heal. Fit. J., vol. 18, no. 5, pp. 11-16, 2014, doi: 10.1249/FIT.000000000000006 7.

[38] M. Cao and M. Quan, "E ff ect of High-Intensity Interval Training versus Moderate-Intensity Continuous Training on Cardiorespiratory Fitness in Children and Adolescents : A Meta-Analysis," 2019.

[39] M. R. A. Razak, E. Ismail, and A. Y. A. Bakar, "Profiling of physical activity, health fitness (Vo2max), body composition, and dietary intake among malaysian university students: A case study," Int. J. Hum. Mov. Sport. Sci., vol. 8, no. 6, pp. 413-420, 2020, doi: 10.13189/saj.2020.080613. 